\title{
Megamaser Activity in Active Galaxies
}

\author{
Willem A. Baan \\ Netherlands Foundation for Research in Astronomy \\ Westerbork Observatory, 7990 AA Dwingeloo, The Netherlands
}

\begin{abstract}
Megamaser emission provides an unprecedented view of the inner nuclear regions of active galaxies. The characteristics of $\mathrm{OH}, \mathrm{H}_{2} \mathrm{CO}$, and $\mathrm{H}_{2} \mathrm{O}$ sources identify different physical environments in the nuclei of megamaser host galaxies. Various classification schemes suggest a dominant starburst nature for the $\mathrm{OH}$ and $\mathrm{H}_{2} \mathrm{CO}$ sources providing the NIR/FIR radiation field as a pump. The molecular emission originates in a 50-100 parsec disk/torus, which is not yet completely observed. For $\mathrm{H}_{2} \mathrm{O}$ masers in relatively dust-free LINER/Seyfert 2 sources, the AGN provides the $\mathrm{X}$-ray radiation field for pumping in a compact sub-parsec thin disk.
\end{abstract}

\section{General Characteristics of Megamasers}

Studies of megamaser emission allow a close-up look at molecular material inside the active nuclei at unprecedented small scales and provide diagnostic tools of their physical environment and dynamics. The different excitation conditions for the masering molecules $\mathrm{OH}, \mathrm{H}_{2} \mathrm{CO}, \mathrm{CH}$, and $\mathrm{H}_{2} \mathrm{O}$ ensure that significantly different nuclear environments are observed.

The initial scenario for $\mathrm{OH}$ megamasers involving relatively low gain and mostly unsaturated amplification of the radio continuum still applies for $\mathrm{OH}$ and $\mathrm{H}_{2} \mathrm{CO}$ sources but less so for $\mathrm{H}_{2} \mathrm{O}$ sources (see Baan et al. 1982; Baan 1997). Optical depths with a significant range between zero and a few (or many) would be expected because of the varying optical depths resulting from imperfect superposition of radio continuum and molecular clouds.

The connection between megamaser activity and the nature of the galactic nuclei has now clearly been established (see Baan 1985 for early evaluation). $\mathrm{OH}$ megamasers are clearly correlated with (mostly) edge-on luminous FIR sources and are pumped by the FIR radiation field. The formaldehyde masers follow the same FIR population but with less stringent FIR temperature criteria. The $\mathrm{H}_{2} \mathrm{O}$ megamasers occur in LINER/Seyfert 2 nuclei.

The relatively low finding rates for $\mathrm{OH}$ and $\mathrm{H}_{2} \mathrm{O}$ megamasers among selected samples result in part from the non-isotropic emission pattern due to the spatial distribution of the amplifying molecular gas. If this rate is solely due to an axisymmetric molecular torus distribution, the detection rates would tell us about the opening angle and size of the molecular structure. The opening angles for such disk geometries have been presented in Figure 1 for $\mathrm{OH}$ and $\mathrm{H}_{2} \mathrm{O}$ sources (Baan 1997). $\mathrm{OH}$ and $\mathrm{H}_{2} \mathrm{CO}$ sources have thick molecular structures and the 


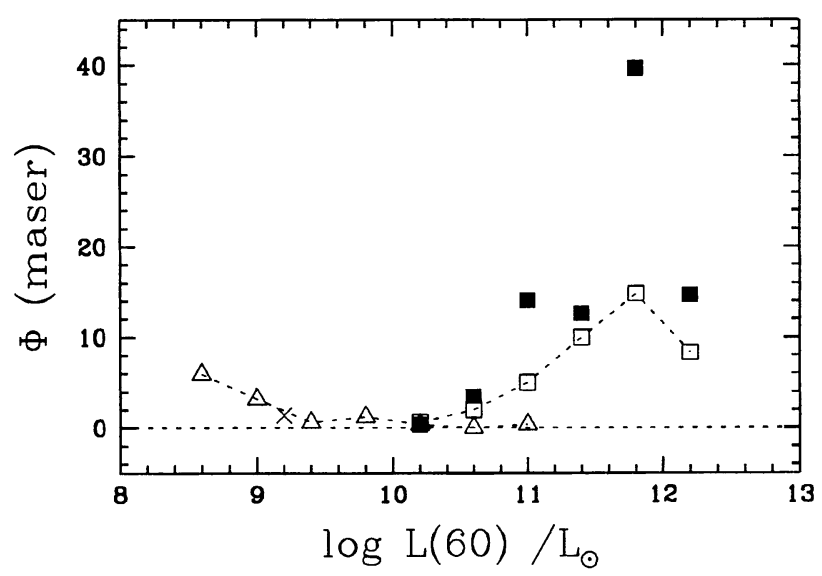

Figure 1. The effective opening angle of the emission pattern for $\mathrm{OH}$ and $\mathrm{H}_{2} \mathrm{O}$ megamaser galaxies. The open (connected) and filled (unconnected) squares for $\mathrm{OH}$ follow from the probability of detecting megamasers among FIR sources using two different luminosity functions. The open triangles for $\mathrm{H}_{2} \mathrm{O}$ follow from the probability of finding masers among LINER/Seyfert 2 sources (Baan 1997).

radial distance of the amplifying material is estimated to be $50-100 \mathrm{pc}$ (Henkel et al. 1987). The $\mathrm{H}_{2} \mathrm{O}$ disks are very thin as has been verified for NGC 4258 (Myoshi et al. 1994).

In the present paper, we will address some megamaser issues that have direct bearing on the nature of the nuclear activity and the spatial characteristics of the molecular emission components. General properties of megamasers have been discussed elswhere (Baan 1991, 1997; Henkel, Baan \& Mauersberger 1993).

\section{Compact Disks of $\mathrm{H}_{2} \mathrm{O}$ Megamasers}

The emission regions in a few powerful and well-studied $\mathrm{H}_{2} \mathrm{O}$ megamaser sources are confined to Keplerian disks (see Haschick et al 1994; Myoshi et al. 1995). The radial distance of the masering cloud within the thin compact disk in NGC 4258 is approximately $0.13 \mathrm{pc}$, while for NGC 1068 the emitting section has an inner radius of $1.3 \mathrm{pc}$ (Gallimore et al. 1996). The likely pumping agent for the $\mathrm{H}_{2} \mathrm{O}$ masers is the X-ray radiation field from the nucleus (Neufeld et al. 1994). For the limited sample of $\mathrm{H}_{2} \mathrm{O}$ sources, the line luminosity is found to be correlated with the product of the X-ray and the radio continuum luminosities suggesting that both radiation fields are of interest (Baan 1997; Braatz et al. 1997). The water-vapor masers provide a high-resolution probe of the nuclear environment of relatively quiescent and low luminosity but "naked" (without much dust) AGNs. 


\section{Optical and Radio Classification of $\mathrm{OH}$ Megamasers}

$\mathrm{OH}$ megamasers are a dominant sub-class of the (super-) luminous FIR galaxies, which at high luminosity show mostly optical AGN characteristics and are in part inter-acting systems. At lower FIR luminosities the sample is predominantly composed of starburst (SBN) nuclei. Optical classification studies of the $\mathrm{OH}$ megamaser sample support this general trend with $45 \%$ showing AGN and with $22.5 \%$ Composite (AGN+SBN) characteristics; the other $32.5 \%$ shows pure starburst characteristics (Baan, Salzer \& LeWinter 1998). On the other hand, the radio classification of the same sample suggests that a majority of $74.5 \%$ shows no or only weak AGN characteristics and resembles radio SBNs (Baan et al 1999). A total of $51 \%$ of the sample show no sign at all of radio AGN activity, which is in agreement with earlier radio surveys of luminous FIR galaxies (Condon et al. 1991). The discrepancy between the optical and radio classifications of $\mathrm{OH} / \mathrm{H}_{2} \mathrm{CO}$ megamasers suggests a uniqueness of luminous FIR sources regard to several characteristics.

(1) In comparison the radio characteristics of the nuclei of $\mathrm{OH}$ and $\mathrm{H}_{2} \mathrm{O}$ sources are found to be quite different. The $\mathrm{OH}$ sources have mostly radio-quiet nuclei while only three sources have outstanding radio-loud sources. Earlier studies suggest that such radio-loud sources are powered by AGNs, while these radio-quiet sources are powered by starbursts (see Helou et al. 1985). In comparison, the majority of the $\mathrm{H}_{2} \mathrm{O}$ nuclei are more radio-loud but they also have lower radio and FIR luminosities than the $\mathrm{OH}$ sources.

(2) A principal compromise picture for luminous FIR sources is a combination of a circumnuclear starburst and a central AGN. The relative activity level of these two components would then determine its outward appearance. However, the locus of LINER/Seyfert 2 sources in the [O III]/H $\beta$ versus $[\mathrm{S} \mathrm{II}] / \mathrm{H} \alpha$ diagnostic diagrams for the $\mathrm{OH}$ megamaser sample (Baan et al. 1998) do not represent a simple mixing curve of two luminosity (AGN + SBN) components. In this alternative picture, the radio and FIR luminosities would be dominated by the (more extended) starburst, while the nuclear optical emission is still dominated by the more obscured AGN component. Several lines of argument could support this picture but it still leaves some unanswered questions.

(3) Large concentrations of molecular gas and dust will affect the optical emissions and diagnostic line ratios, because different line emissions originate at different radial locations and at different obscuration depths within the galaxy. For instance, the majority of the $\mathrm{OH}$ sources have (buried) edge-on dust lanes, giving high values of $A_{V}$ for the emission from the nucleus itself but lower $A_{V}$ for emissions from the outlying regions above and below the plane.

(4) A mixture of starburst and AGN activity has also been proposed on the basis of recent ISO data. An infrared "diagnostic diagram" based on the ratio of high- and low-excitation mid-IR emissions lines versus the strength of the $7.7 \mu \mathrm{m}$ PAH feature shows that the ultra-luminous FIR galaxies form a distinct AGN - SBN "mixing sequence". (Genzel et al. 1998). About $70 \%-80 \%$ of the luminous FIR galaxies are predominantly powered by starbursts rather than by AGNs, which is very consistent with the radio classification for megamaser galaxies (Baan et al. 1999). For example, several prominent $\mathrm{OH}$ megamasers IR17208, IR12112, and Arp 220 (see section below) which have LINER/Seyfert 2 optical spectra fall in this group in the radio and according to the infrared 


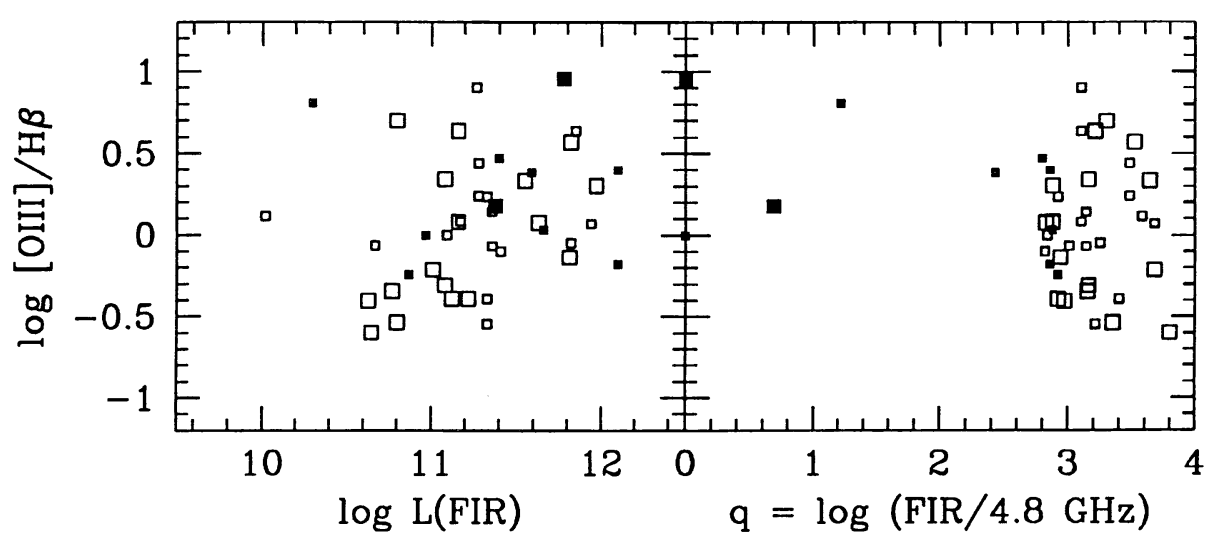

Figure 2. The relation between the optical diagnostic $[\mathrm{O}$ III $] / \mathrm{H} \beta$ ratio for $\mathrm{OH}$ megamasers and (a) their $60 \mu \mathrm{m}$ FIR luminosity and (b) their "q-ratio" of the FIR luminosity and $4.8 \mathrm{GHz}$ radio continuum luminosity. The galaxies have been marked to indicate their radio characteristics as follows: (1) large open squares show no evidence of AGN activity, (2) small open square show minimal AGN activity, (3) small filled squares show moderate AGN activity, and (4) large filled squares show strong AGN characteristics (Baan et al. 1998; 1999).

"mixing sequence" (Genzel et al. 1998). We find agreement between the nuclear classification in the radio and the infrared but we also find that the optical classifications can be discrepant from the other two.

(5) The combination of a modified mass function for the nuclear starburst with a higher mass cutoff and of superwinds emanating from the region could somewhat harden the ionizing radiation field and consequently change the optical emission line ratios. Nuclear superwinds (Heckman et al. 1990) and $\mathrm{OH}$ molecular outflows showing outflow velocities up to $1000 \mathrm{~km} \mathrm{~s}^{-1}$ (Baan et al. 1987) provide a consistent picture of a general blow-out in galaxies undergoing massive central starbursts.

(6) A critical parameter in the optical classification is the $[\mathrm{O}$ III] $/ \mathrm{H} \beta$ ratio, which increases significantly when moving from starburst nuclei to LINERs and to Seyferts. In Figure 2 the optical line ratio has been plotted against the 60 $\mu \mathrm{m}$ FIR luminosity and against the "q-ratio" of FIR luminosity over the $6 \mathrm{~cm}$ radio luminosity (see Baan et al. 1998; 1999). Figure 2(a) shows a roughly linear increase of $[\mathrm{O}$ III] $/ \mathrm{H} \beta$ with the FIR luminosity, which could suggest that the generation of optical emission and FIR emission is due to the same process independent of its optical classification. Frame 2(b) shows that only two AGN galaxies stand out clearly with a low q-value characteristic of AGNs; most of the galaxies have a q-ratio in the range characteristic of SBNs.

The above information suggests that contrary to this optical line characteristics, the majority of megamaser galaxies are indeed predominantly powered by nuclear starbursts. Due to a variety of circumstances the intense starburst re- 
gion in a (super-)luminous FIR/OH galaxy appears to mimic radio-quiet AGNs in their optical appearance. If AGNs were present in these nuclei, they must be radio-quiet but optical-loud. This result implies that the standard optical classification scheme for these high activity super-luminous galaxies may not be accurate enough as a diagnostic.

\section{OH Emission Structure of III Zw35}

The $\mathrm{OH}$ megamaser III Zw35 has been studied with MERLIN (Montgomery $\&$ Cohen 1992) and more recently with the VLBA (Trotter et al. 1997). The galaxy exhibits several extended emission regions with a total extent of $50 \mathrm{pc}$. At high resolution the more extended emission regions breaks into compact maser features, which together account for about 50 percent of the flux in the double peaked $\mathrm{OH}$ emission profile. The distribution of the $\mathrm{OH}$ emission and the fact there is missing flux support the idea that the maser features originate in a ring/torus centered on the nucleus of III $\mathrm{Zw} 35$. One could argue that the two main emission regions at 48 parsec separation and $100 \mathrm{~km} \mathrm{~s}^{-1}$ velocity separation signify the tangential and frontal regions of a Keplerian torus. If this were true, the nuclear mass needs to be $1.4 \times 10^{7} \mathrm{M}_{\odot}$. The rest of the missing flux should also come from this torus. The inferred radial distance of the masering clouds in the torus is also consistent with FIR pumping models (Henkel et al. 1987; Kylafis \& Pavlakis 1998).

\section{5. $\mathrm{OH}$ and Radio Continuum Structure of Arp 220}

The prototype megamaser galaxy Arp 220 (IC 4553) has its most prominent $\mathrm{OH}$ and $\mathrm{H}_{2} \mathrm{CO}$ emission at the western nucleus and less prominent emission at the eastern nucleus (Baan \& Haschick 1995). Spectral line VLBI studies with the EVN reveal three high brightness $\mathrm{OH}$ components: two components south of the eastern radio nucieus separated by $40 \mathrm{pc}$ and one north of the western radio nucleus (Diamond et al. 1990; see Baan \& Haschick 1995). These components appear not to coincide with the radio nuclei themselves as predicted for any compact AGN - nuclear torus scenario. Very recent VLBI results reveal a cluster of compact radio supernovae at both nuclei of Arp 220 (Smith et al. 1998). Two extended $\mathrm{OH}$ emission regions in the western nucleus straddle the cluster of supernova remnants and could together form a north-south torus structure with radius of $45 \mathrm{pc}$ (Lonsdale et al. 1998). At the eastern nucleus two components also straddle the cluster but lie slightly south of the radio peak. However, the emission found (to date) at the eastern nucleus is not at the nuclear velocity and thus unrelated to the nucleus itself (see paragraph below). The VLBI features found at the nuclei account for about $40 \%$ of the single dish flux. More diffuse emission must be present in the intervening regions, particularly at the western nucleus.

Also the $\mathrm{H}_{2} \mathrm{CO}$ emission also comes predominantly from the western nucleus with only weak emission from a double north - south structure southeast from the eastern nucleus and from some more intervening regions between the two nuclei (Baan \& Haschick 1995). Recent NIR images of Arp 220 made with NICMOS (Scoville et al. 1998) show an emission structure almost identical to 
that of the $\mathrm{H}_{2} \mathrm{CO}$ emission. Such coincidences suggest that (1) obscuring dust plays havoc in this region and that large fractions of the nuclear regions are heavily obscured, and that (2) the formaldehyde and $\mathrm{OH}$ masers each appear to be pumped by the FIR/NIR radiation fields.

A simple orbital model has been proposed for the dynamics of the two nuclei based on molecular line characteristics (Baan \& Haschick 1995; Scoville et al. 1996). Both nuclei show $\mathrm{OH}$ emission closely to $5300 \mathrm{~km} \mathrm{~s}^{-1}$, which may be due to foreground material at the eastern nucleus. The $\mathrm{OH}$ features at the systemic velocity of the eastern nucleus are to be found in the second spectral feature, which is in fact a mixture of the $1667 \mathrm{MHz}$ lines from the eastern nucleus and the $1665 \mathrm{MHz}$ lines from the western nucleus (see Baan \& Haschick 1987).

\section{High Redshift $\mathrm{OH}$ Sources}

Megamaser searches of galaxies at high radial velocities have in the past been severely limited by receiver capabilities and spectral coverage. Only for $\mathrm{OH}$ it has been possible to explore higher redshift regimes. The formaldehyde sources found to date are too weak to be detectable much beyond the local universe. On the other hand, the $\mathrm{H}_{2} \mathrm{O}$ megamasers are very powerful at lower redshifts but their detection rate has been very low. Again the $\mathrm{H}_{2} \mathrm{O}$ searches at higher redshifts will be severely limited by the receiver capabilities at the larger telescopes.

Powerful $\mathrm{OH}$ megamasers have now been found to redshifts of about $\mathrm{z}=$ 0.26 or $\mathrm{v}=80,000 \mathrm{~km} \mathrm{~s}^{-1}$, which is similar to those of luminous (thermal) $\mathrm{CO}$ sources. If $\mathrm{OH}$ megamaser activity is rather common locally, $\mathrm{OH}$ emission may be found in the very red objects in the range $z=1-2$, where the population of quasars peaks. With a total velocity width of $2400 \mathrm{~km} \mathrm{~s}^{-1}$, the currently highest redshift $\mathrm{OH}$ maser IRAS $14070+0525$ suggests that the dynamic structure of such sources may be quite different from those observed at lower redshifts (Baan et al. 1992). Although there may be a violent starburst or merging activity in this source, the radio and optical images do not yet reveal any interaction or merger nature.

A quadratic luminosity relation observed for the $\mathrm{OH}$ sources may render $\mathrm{OH}$ megamasers observable to very high redshifts. For current observing systems, a megamaser is required to have $\mathrm{L}_{O H}=10^{3.8} \mathrm{~L}_{\odot}$ to be observable at $\mathrm{z}=2$; already two nearby masers have been detected with such luminosities. Calculations of the number of detectable $\mathrm{OH}$ sources suggest that the observable universe increases faster than the decrease in the spatial density of $\mathrm{OH}$ galaxies, and that at redshifts $\mathrm{z}=1-2$ large numbers of detectable sources are expected (Baan 1991; Briggs 1998). The OH detection rate at high redshifts will depend on the actual $\mathrm{L}(\mathrm{FIR})-\mathrm{L}(\mathrm{OH})$ relation and on the galaxy merger rate, that plays a significant role in making the population of luminous FIR sources (see Briggs 1998). The $\mathrm{OH}$ detection rate will also depend strongly on the sensitivity of the observing systems favoring telescopes such as the upgraded Arecibo and the to-be-built Square Kilometer Array (SKA). Probing the higher redshift ranges for $\mathrm{OH}$ activity will provide independent tests of cosmological models and the nuclear evolution of galaxies. 


\section{Conclusions}

Radio and molecular line studies of megamasers provide independent probes of the nuclear environment and the dynamics of active galaxies. The compact disks in $\mathrm{H}_{2} \mathrm{O}$ sources tell us about the physics of an AGN and the nuclear radiation environment. The torus/ring structures in $\mathrm{OH}$ and $\mathrm{H}_{2} \mathrm{CO}$ sources provide an excellent probe of the critical circumnuclear environment that shapes the (super)luminous FIR galaxies. The missing flux in both III Zw35 and Arp 220 likely come from lower brightness (maser) emission regions distributed along the dusty torus.

The radio and NIR classifications of luminous OH/FIR sources largely agree on a dominant starburst powering these galaxies rather than an AGN, although the optical classification may still suggest an AGN nature. At high redshift no clear connection is yet available between $\mathrm{OH} / \mathrm{H}_{2} \mathrm{CO}$ activity and a particular part of the galaxy population. Possibly the very red objects found at higher redshift or even type 2 quasars are the megamaser host galaxies. If so, continued molecular studies will provide valuable new insights into the nature of such peculiar objects.

\section{References}

Baan, W.A. 1985, Nature, 315, 26

Baan, W.A. 1989, Ap.J., 338, 804

Baan, W.A. 1991, in Skylines, Third Haystack Observatory Conference, A.D. Haschick and P.T. Ho, P.A.S.P. Conf. Series, 16, 45

Baan, W.A. 1997, in High Sensitivity Radio Astronomy, R.J. Davis \& N.J. Jackson, Cambridge University, 73

Baan, W.A. \& Haschick, A.D. 1987, Ap.J., 318, 139

Baan, W.A. \& Haschick, A.D. 1995, Ap.J., 454, 745

Baan, W.A., Haschick, A.D. \& Uglesich, R. 1993, Ap.J., 415, 140

Baan, W.A., Henkel, C. \& Haschick, A.D. 1987, Ap.J., 320, 154

Baan, W.A. et al. 1992, Ap.J., 396, L99

Baan, W.A., Kloeckner, H.-R., Haschick, A.D., \& Besenfelder, E. 1999, Ap.J., submitted

Baan, W.A., Salzer J.J., \& LeWinter, R.D. 1998, Ap.J., December 15

Baan, W.A., Wood, P.A.D. \& Haschick, A.D. 1982, Ap.J., 260, L49

Braatz, J., Wilson, A.S., \& Henkel, C. 1997, Ap.J. Suppl., 110, 321

Briggs, F.H 1998, Astron. Ap.,

Condon, J.J, Huang, Z.-P., Yin, Q.F., \& Thuan, T.X. 1991, Ap.J., 378, 65

Diamond, P., Norris, R. Baan, W.A., \& Booth, R. 1990, Ap.J., 340, L49

Gallimore, J., et al. 1998, Ap.J., 462, 740

Genzel, R. et al. 1998, Ap.J., 498, 579

Haschick, A.D., Baan, W.A., \& Peng, E. 1994, Ap.J., 437, L35

Heckman, T.M., Armus, L., \& Miley. G.K. 1990, Ap.J. Suppl., 74, 833 
Henkel, C., Baan, W.A., \& Mauersberger, R. 1993, Astron. Ap. Rev., 3, 47

Henkel, C., Güsten, R. \& Baan, W.A. 1987, Astron. Ap., 185, 14

Helou, G., Soifer, B.T., \& Row, R.M. 1985, Ap.J., 298, L11

Kylafis, N.D. \& Pavlakis, K.G. 1998, IAU Highlights of Astronomy, 23, in press

Lonsdale, C.J., Lonsdale, C.J., Diamond, P.J., \& Smith, H.E. 1998, Ap.J., 493, L13

Montgomery, A.S. \& Cohen, R.J. 1992, M.N.R.A.S., 254, 23p

Myoshi, M. et al. 1995, Nature, 373, 127

Neufeld, D., Maloney, P., \& Conger, S. 1994, Ap.J., 436, L127

Scoville. N.Z., Yun, M \& Bryant, P. 1997, Ap.J., 484, 702

Scoville, N.Z. et al. 1998, Ap.J., 492, L107

Smith, H.A, Lonsdale, C.J., Lonsdale, C.J., \& Diamond, P.J. 1998, Ap.J., 493, L17

Trotter, A.S. et al. 1997, Ap.J., 485, L79 